\title{
Exercise-induced Improvements in Cognitive Functioning and Brain Structure in Older Adults
}

\author{
Sara Bartel*
}

\begin{abstract}
Physical activity is often associated with benefits such as reduced risk of diabetes and cardiovascular disease; however, the benefits of physical activity are not only limited to physical health, but also extend to cognition (Warburton, Nicol \& Bredin, 2006). Exercise in older populations results in improved cognitive functioning and decreased risk of cognitive decline (Muscari et al., 2010). This current literature review examines the association between physical activity and cognitive functioning in older adults. Studies suggest that a variety of types of exercise have cognitive benefits, although it is not clear which type of activity has the largest effect. Additionally, exercise increases total brain volume and the connectivity of neural networks in areas such as the hippocampus, and decreases the presence of white matter hyperintensities in areas involved in motor control and coordination (Erickson et al., 2010; Tseng et al., 2013). Taken together, the research indicates that physical activity improves cognitive functioning and causes exercise-induced changes in the brain. Limitations of the reviewed research include a lack of generalizable results due to a lack of diversity of samples, as well as the presence of cross-sectional designs that are unable to define the direction of the relationship between exercise and cognition. Future research should investigate the inconsistencies reported in the literature with the goal of developing programs to improve cognitive function in older populations and to reduce the burden of cognitive impairment on the health care system.
\end{abstract}

Keywords: exercise, cognitive functioning, brain structure, older adults

Physical activity, in a variety of forms, is often associated with a reduced risk of mortality, cardiovascular disease, diabetes mellitus and cancer, as well as an increase in psychological well being (Warburton, Nichol \& Bredin, 2009). In addition to these effects for individuals of all ages, physical activity is also associated with further physical benefits for older populations. In such populations, exercise is linked to increased bone density and a lower risk of falls, two factors that are tend to decreases rates of mortality
(Mussolino \& Gillum, 2008; Rubenstein, 2006; Vogel et al., 2009).

Physical activity is most commonly associated with the aforementioned physical and psychological benefits; however, the benefits of physical activity also extend to cognition. Exercise results in improved cognitive functioning and brain health for individuals of all ages. In childhood, aerobic exercise has beneficial effects on perceptual skills, math and verbal ability, as well as IO

*Department of Psychology, College of Arts and Science, University of Saskatchewan, Saskatoon, SK, Canada

Correspondence: sjb7og@mail.usask.ca 
(Sibley \& Etnier, 2003). These benefits continue into young adulthood where aerobic exercise has been shown to increase factors such as cognitive control, cognitive flexibility, the speed of response, and the speed of visual search (Stroth et al., 2010; Tomporowski, 2003). In older adults the benefits of exercise continue, but they have even more significant implications.

Beginning at middle age, or occasionally even earlier, cognitive abilities such as reasoning, processing speed and memory begin to decline (Deary et al., 2009). This is especially true for executive function (EF), an umbrella term for many higher-order psychological processes such as inhibition, working memory and cognitive flexibility which are the building blocks of planning, problem solving and reasoning (Deary et al., 2009; Diamond, 2013; Rasmussen, 2005). Together, EF processes are involved in consciously engaging in problem solving and behaviors to achieve a certain goal (Rasmussen, 2005; Song \& Hakoda, 2014). In order to measure $E F$ and to separate it from non-EF cognitive functions such as alertness, sustained attention, divided attention and visual scanning, the Stoop Test and Wisconsin Card Soring Test are often used (Grant \& Berge, 1948; Song \& Haokda, 2014).

The decline of cognitive processes that occurs as adults age is inherently tied to structural changes in the brain. Older adults often demonstrate a decrease in the white matter integrity and total volume of the brain, which results in a decline in cognitive abilities such as working memory (Deary et al., 2009). Physical activity appears to have a protective effect and can reduce or reverse these age-related declines at both the functional and neurological level. Rovio et al. (2005) suggests that the protective effect of exercise may occur through a variety of mechanisms. Exercise reduces vascular risk factors (i.e. diabetes, hypertension and being overweight) that have been demonstrated to play a role in the development of both dementia and Alzheimer's diseases (Rovio et al., 2005). Additionally, exercise plays a protective role through neurobiological mechanisms (Rovio et al., 2005). Exercise has been found to increase growth factors, stimulate neurogenesis, and result in the activation of processes that likely promote brain plasticity in animal models, all of which are tied to improved cognitive functioning (Cotman \& Berchtold, 2002; Rovio et al., 2005). Moreover, physical activity also impacts neruotrophic factors and gene transcripts that are involved in the maintenance of cognitive functioning (de Melo Coelho et al., 2013; Rovio et al., 2005). Finally, Rovio et al. (2005) propose that exercise may be protective through socioeconomic and lifestyle factors that increase the likelihood of leisure-time physical activity. This additional leisure-time physical activity increases the chance for exercise to serve a protective function. These potential pathways suggested by Rovio et al. (2005) are associated with the concept of cognitive reserve.

Cognitive reserve refers to how flexibly and efficiently an individual can optimally use the theoretical capacity or upper limit of the brain and involves compensating for damage or other pathologies by using the brain more effectively or recruiting alternate networks (Stern, 2002; Tucker \& Stern, 2011). The cognitive reserve hypothesis suggests that greater cognitive reserve is associated with better stability of cognitive function during advancing age or brain damage (Etnier, 2009). Physical activity is associated with increased cognitive reserve; exercise better equips individuals to initiate neuroprotective processes in response to neurodegenerative processes, as well as other forms of neural dysfunction to develop during adulthood (Nithianantharajah \& Hannan, 2009). This suggests that the protective effect of physical activity on cognitive processes and brain functioning is linked to increased cognitive reserve (Etnier, 2009).

\section{Literature Review}

This literature review seeks to investigate the nature of the relationship between exercise and cognitive functioning in older adults. Research based on resistance training, cardiovascular endurance and combined interventions will be reviewed. Additionally, several studies examining the exercise-induced changes in the brain via imaging methods will also be discussed to connect the changes in cognitive performance associated with exercise to changes in neurological structure and function.

\section{Resistance Training}

Resistance training involves repetitive exercises with free weights, body weight or exercise machines and is aimed at improving muscle strength. In a 24-week resistance training intervention, Cassilhas et al. (2007) divided a group of males into moderate and high intensity resistance training groups, as well as a stretching control group. All groups were given the Rey-Osterrieth complex figure test to assess long-term episodic memory, the Toulouse-Peron's concentration attention test to assess attention, the Weschler Adult Inellgience Scale III to assess short-term memory, and the Wechsler Memory ScaleRevised to assess visual short-term memory (Cassilhas et al., 2007). Both resistance training groups improved on a variety of cognitive functions including short-term memory, long-term memory, attention, and EF in comparison to the stretching control group (Cassilhas et al., 2007). Considering both levels of intensity of resistance training resulted in similar improvements, the results suggest that once intensity level reaches a point, increases in intensity do not have further benefits for cognitive functioning. 
Resistance training interventions have also been associated with increased performance on measures of EF. In a four-week intervention of resistance training, Anderson-Hanley, Nimon and Westen (2010) found that individuals in the resistance training group improved on two measures of EF - the Stroop C and the Digit Span Backwards. In a similar study, Liu-Ambrose et al. (2010) reported that after one year, a group of women who engaged in resistance training once or twice a week significantly improved on measures of conflict tasks and selective attention, both subsumed under the umbrella of EF. At a follow-up assessment one year after the cessation of training, the authors found that individuals who had taken part in the resistance training intervention once a week had further improved their performance on conflict resolution and selective attention by $15 \%$ in comparison to the control group (Davis et al., 2010). Despite this, the women who had engaged in resistance training twice a week did not maintain their cognitive benefits (Davis et al., 2010). This lack of maintenance may be that these women had, on average, a $6.6 \%$ decrease in physical activity level from their baseline measure, while the individuals who engaged in resistance training once a week had a $5 \%$ increase in physical activity level from their baseline measure (Davis et al., 2010). This would suggest that maintaining physical activity level is essential to maintaining the cognitive benefits of resistance training, which appear to primarily relate to EF.

\section{Aerobic and Cardiovascular Training}

Improvement in EF has also been found in aerobic and cardiovascular training programs. In a study by Albinet, Boucard, Bouquet and Audiffen (2010), individuals participated in an aerobic training program that included walking, circuit-training and running. These activities, designed to increase aerobic endurance and cardiovascular fitness, significantly increased the participants EF performance on the Wisconsin card sorting test in comparison to the control group (Abinet et al., 2010). Furthermore, the aerobic group also increased their heart rate variability, which is a measure of the cardiovascular autonomic function related to efficient cognitive processing (Abinet et al., 2010).

Despite this significant finding, no effect of aerobic exercise was found on EF in a study of older Japanese adults conducted by Hatta, Nishihira, and Higashiura (2013). It may be that this differing result occurred because participants only engaged in a single bout of walking conducted at their own pace. This intervention was most likely not long enough to have any benefits. Similarly, Oken et al. (2006) also failed to find any improvements in EF due to aerobic exercise. This non-significance may suggest that $E F$ is unaffected by aerobic exercise, or it may have been due to the inclusion factors of the study, which allowed individuals who exercised up to 210 minutes per week to participate. As 210 minutes is over the weeklyrecommended amount of exercise, the training sessions may not have involved more physical activity than usual, thus not providing anything from which the participants could gain benefits (World Health Organization, 2015). Although this is a limitation of the study, the results are consistent with the results of Marmeleira, Godinho and Fernandes (2009). The researchers also failed to find an effect of exercise on EF; however, they did find improvements in behavioral speed and visual attention in an aerobic training group (Marmeleira et al., 2009).

The relationship between EF and aerobic exercise is not clear. Although Albinet et al. (2010) found improvement, this finding is not shared with other studies. It is possible that aerobic exercise only increases EF when the activity involves substantial exertion. For example, participants in Albinet et al. (2010) took part in walking, circuit training and running, while those participants in the aerobic training groups in the studies by Hatta et al. (2013), Oken et al. (2006) and Marmeleria et al. (2009) only participated in walking activities, which can be assumed to be less strenuous. Future research should investigate and clarify this relationship.

Aerobic endurance training is also associated with reduced rates of cognitive decline. Muscari et al. (2010) placed older adults into a control group or a 12-month endurance training program that included treadmill, bicycle and free body activity. At the end of the study the individuals in the endurance training program either maintained or improved their performance on the minimental state examination (MMSE), which measures orientation (awareness of time and place), registration (immediate memory), recall, language and attention and calculation (Muscari et al., 2010). In contrast, the individuals in the control group worsened in language, orientation and attention and calculation in comparison to their baseline scores (Muscari et al., 2010). These results suggest that endurance training has a protective effect over age-related decline of cognitive functioning.

\section{Contradictions in Resistance Training and \\ Aerobic Training}

Although both resistance training and aerobic training have been found to effectively increase components of cognitive functioning, the results of research including both resistance training and aerobic training groups are inconsistent. Smiley-Oyen, Lowry, Francois, Kohut and Ekkekakis (2008) separated 57 older adults into a cardiovascular group (consisting of aerobic training) and a flex-tone group (consisting of strength, flexibility and resistance training). In both groups, physical activity did not 
increase performance on cognitive tasks requiring little $E F$; however, the cardiovascular group did significantly reduce their reaction time and their number of errors on an EF task, which is indicative of improved EF performance (SmileyOyen et al., 2008). In contrast, no such improvements were found for the flex-tone group (Smiley-Oyen et al., 2008). This result suggests that aerobic training may have a more beneficial effect on EF than resistance training. Despite this suggestion, the results of Nagumatsu, Handy, Hsu, Voss and Liu-Ambrose (2012) are inconsistent with this finding. Instead of finding benefits from an aerobic training, the authors reported that resistance training resulted in significant improvement on measures of EF and associative memory, while aerobic exercise had no effect on cognitive functioning (Nagumatsu et al., 2012). The authors commented that the aerobic group had the highest rate of attrition, which could be one possible explanation for the lack of improvement in cognitive functioning in the group. Taken together, the results of these two studies demonstrate the inconsistent nature of research conducted on this topic.

\section{Combination Training}

To take advantage of the effects of resistance training and aerobic training and to create a more cohesive perspective of the effects of exercise on cognitive functioning, many researchers combine resistance training and aerobic training into one intervention. Williamson et al. (2009) compared the results of two groups - a group that engaged in aerobic walking, as well as flexibility, balance and strength exercises, and an active control group who received a health education program. As this study was only designed to be a pilot program and not designed to detect significant differences in cognitive functioning between the two groups, the researchers used correlation in their analysis. Improvements in psychomotor speed and working memory were found to be positively associated with the combined physical activity group (Williamson et al., 2009). This finding aligns with the results of Brown, Liu-Amrbose, Tate and Lord (2009) who conducted a study with a similar intervention. The researchers found that exercise significantly improved fluid intelligence, a component of cognition strongly related to working memory (Brown et al., 2009; Fukuda, Vogel, Mayr \& Awh, 2010).

\section{Exercise-Induced Changes in the Brain}

Physical activity is associated with increased total volume, grey matter and white matter volume, as well as increased connectivity and reduced white matter hypertensities (white matter lesions). These neurological changes are associated with increased functioning across a variety of domains of cognitive abilities. In an investigation of neurological changes, Rovio et al. (2010) found that in comparison to individuals who were sedentary at midlife, individuals who engaged in physical activity at least twice a week at midlife had a greater total brain volume and a greater grey matter volume in later life. Additionally, the active individuals had greater grey matter density in the middle frontal gryus, an area related to EF (Rovio et al., 2010). Rovio et al. suggest that this area may have more density because it is located in close proximity to the motor cortex, which is active during physical activity. As a result of this activation, the density of brain areas that are closely related to the motor cortex (such as the middle frontal gyrus) may be preserved or improved (Rovio et al., 2010).

In a similar study, conducted by Benedict et al. (2013) physical activity level was positively associated with total brain volume and white matter volume. Moreover, the researchers also report a positive association between physical activity level and grey matter volume in the bilateral precuneus, an area involved with cognitive processes such as visuo-spatial imagery and episodic memory retrieval (Benedict et al., 2013; Cavanna \& Trimble, 2006). Furthermore, Erickson et al. (2010) found that walking 72 blocks a week was positively related to larger volume of grey matter in two areas - the hippocampus, which is involved in spatial memory and transferring shortterm memories to long-term memories, and the entorhinal cortex, which processes information for the hippocampus and contributes to attention and spatial skills (Bird \& Burgess, 2008; Coutureau \& Di Scala, 2009). Although the results of these studies are not causal, and thus may be influenced by a multitude of additional factors, they seem to suggest that physical activity has positive benefits for the overall brain, and most importantly for the areas involved with memory, attention, visuo-spatial functioning, and EF.

In regards to white matter, exercise appears to not only increase its volume but also maintain the structural integrity of white matter. Tseng et al. (2013) reported that in comparison to their sedentary counterparts, older adults who engaged in life long aerobic exercise training had an $83 \%$ reduction in deep white matter hyperintensity volume. Specifically, white matter structural integrity was preserved in the brain networks related to motor control, visuospatial function and coordination (Tseng et al., 2013). This preservation was found to be associated with the level of aerobic fitness, thus suggesting that fitness level is more important than amount of activity (Tseng et al., 2013). Similarly, Johnson, Kim, Clasey, Bailey \& Gold (2012) found a positive correlation between cardiorespiratory fitness and white matter integrity in the interconnecting frontal areas of the corpus callosum involved in voluntary motor tasks and high-level motor planning. This suggests that increased physical activity and related aerobic fitness level may slow down the age-related myelin declines in motor planning (Tseng et al., 2013). 
Research also indicates that physical activity increases connectivity between areas of the brain, a sign of increased speed of information transfer. Burdette et al. (2010) reported that a six-week walking intervention did not change global brain connectivity; however, it did increase connectivity within the hippocampus, as well as between the hippocampus and anterior cingulate gyrus. Voss et al. (2010) also found that walking resulted in increased connectivity within the brain. After one year, those in the walking intervention demonstrated increased connections between the default mode network (anterior prefrontal cortex, tempoparietal junction, dorsal posterior and anterior cingulate gyri, and the insular and frontal operculum cortices) and the frontal executive network (posterior cingulate, ventral and superior frontal medial cortices, middle temporal cortices, and the bilateral occipital, middle frontal hippocampal and parahippocampal) (Voss et al., 2010). This increase in connectivity was so great that the brains of the older adults resembled those of younger adults (Voss et al., 2010). This is an important link, as the default mode network is involved in autobiographical memory and memory consolidation, and the frontal executive network, is involved in error feedback and maintaining relationships between actions and related outcomes (Voss et al., 2010).

\section{Exercise interventions and Brain Function}

The relationship between physical activity and brain volume has also been investigated in the context of exercise interventions. Colcombe et al. (2006) compared older adults in an aerobic group to younger participants. The older adults who engaged in aerobic exercise showed a significant increase in both grey matter and white matter, especially in the areas in the frontal lobes involved with high-level attentional control and memory functions (Colcombe et al., 2006). Importantly, white and grey matter volume also increased in areas in the prefrontal and temporal cortices that often lose volume with age (Colcombe et al., 2006). Additionally, the younger participants did not show a significant increase in volume of grey or white matter, suggesting that the benefits of physical activity on brain volume apply selectively to older adults (Colcombe et al, 2006). It is not clear what benefits these exercise-induced changes in the brain truly have on cognition because Colcombe et al. (2006), like many other researchers in this field, did not use any measures of cognitive abilities or overall cognitive functioning.

Despite this lack of inclusion by Colcombe et al. (2006), both Mortimer et al. (2012) and Erickson et al. (2011) did include measures of cognitive functioning and found direct connections among physical activity, cognitive functioning and neurological changes. Erickson et al. (2011) reported a $1.97 \%$ increase in the volume of the right hippocampus and a $2.12 \%$ increase in the volume of the left hippocampus following an aerobic training program. The authors stated that this increase in the bilateral hippocampus was directly related to increased performance in spatial memory (Erickson et al., 2011). In contrast to the aerobic group, the control group had a $1.40 \%$ decline in volume in the left hippocampus and a $1.43 \%$ decline in the volume of the right hippocampus over the same time period (Erickson et al., 2011). Such age-related shrinkage of the hippocampus has been associated with memory impairments and an increased risk of developing dementia (Erickson et al., 2011). Thus, aerobic exercise appears to be neuroprotective against both memory and cognitive decline (Erickson et al., 2011).

Mortimer et al. (2012) did not find exercise-induced changes in the brain related to walking, a light aerobic exercise; however, the researchers did report a significant effect of tai chi, a non-aerobic activity involving a variety of slow movements. Tai chi training resulted in a $.45 \%$ increase in total brain volume, as well as an increase in performance on a variety of cognitive abilities including delayed recognition, mental flexibility and EF. Although this result is encouraging, it is not clear how much the sustained attention required by tai chi contributed to the increase in total brain volume.

\section{General Findings}

The literature concerning the relationships between physical activity and cognitive functioning in individuals over age 50 includes many contradictions. While many studies have reported that physical fitness and not the amount or type of physical activity is the most important contributor to cognitive functioning, Smiley-Oyen et al. (2008) and Nagamatsu et al. (2012) reported that a change in cognitive performance was not mediated by changes in aerobic fitness. Additionally, many studies have reported that several factors, such as the type of physical activity, are not as critical as the intensity of physical activity. Furthermore, it appears that although exercise may improve numerous areas of cognitive functioning, physical activity seems to have the greatest number of benefits in relation to EF (Davis et al., 2010). Taken together, the reviewed research suggests that different types of physical activity may result in different benefits for cognitive abilities, all of which may be contingent on the combinations of intensity and amount of activity.

While there is a plethora of literature reviews and meta-analyses examining the topic of exercise and cognition or brain function, there has not been, to my knowledge, a literature review that has combined all of the aspects included in the current review. Moreover, as this literature review only included research published from 
2005 and onwards, it is more recent than many other existing reviews. Additionally, this review only included studies done with cognitively healthy subjects, while many other reviews include or focus on individuals with cognitive decline. Thus, this review offers a novel contribution.

\section{Limitations}

The majority of the reviewed research was conducted with healthy subjects from urban areas who demonstrated relatively high cognitive functioning. Consequently, the results of this research may not be generalizable to other populations. Additionally, many of the samples were predominantly female. Colcombe and Kramer (2003) report that such samples yield stronger effect sizes in studies on the relationship between exercise and cognitive functioning. This implies that certain studies reported in the current literature review would have a reduced effect size if conducted with equal proportion of men and women. Furthermore, a large number of studies on this topic have been conducted with correlational methods or cross sectional designs that are unable to determine the direction of the relationship between physical activity and cognitive function.

\section{Future Research and Implications}

Future research should investigate the inconsistencies reported in the literature to discover which type, amount, or level of intensity of physical activity produces the greatest number of benefits in relation to cognitive functioning and exercise-induced changes in the brain. Research should further consider which types of activity are associated with specific cognitive abilities and determine which areas of cognitive functioning have the potential for the most improvement. Finally, the relationship between sex, exercise and cognitive benefits should be considered to determine whether the benefits of physical activity are stronger for women, and whether many of the results of the reviewed research could be attributed to the ratio of male and female participants. Along with the current findings, this future research would have implications for the health care system, as well as public policy. Exercise programs should be made widely available and should be specifically developed and implemented with the goal of improving cognitive functioning in older populations. Such programs would have the potential to reduce the burden of cognitive impairment on the health care system, as well as on families and caregivers. 


\section{References}

Albinet, C. T., Boucard, G., Bouquet, C. A., \& Audiffren, M. (2010). Increased heart rate variability and executive performance after aerobic training in the elderly. European Journal of Applied Physiology, 109(4), 617-624. doi: 10.1007/s00421-010-1393-y

Anderson-Hanley, C., Nimon, J. P., \& Westen, S. C. (2010). Cognitive health benefits of strengthening exercise for community-dwelling older adults. Journal of Clinical and Experimental Neuropsychology, 32(9), 996-1001. doi: 10.1080/13803391003662702

Benedict, C., Brooks, S. J., Kullberg, J., Nordenskjöld, R., Burgos, J., Le Grevès, M., ... \& Schiöth, H. B. (2013). Association between physical activity and brain health in older adults. Neurobiology of Aging, 34(1), 83-9o. doi: 10.1016/j.neurobiolaging.2012.04.013

Bird, C. M., \& Burgess, N. (2008). The hippocampus and memory: Insights from spatial processing. Nature Reviews Neuroscience, 9(3), 182-194. doi:10.1038/nrn2335

Brown, A. K., Liu-Ambrose, T., Tate, R., \& Lord, S. R. (2009). The effect of group-based exercise on cognitive performance and mood in seniors residing in intermediate care and self-care retirement facilities: A randomized controlled trial. British Journal of Sports Medicine, 43(8), 608-614. doi: $10.1136 /$ bjsm.2008.049882

Burdette, J. H., Laurienti, P. J., Espeland, M. A., Morgan, A., Telesford, Q., Vechlekar, C. D., ... \& Rejeski, W. J. (2010). Using network science to evaluate exerciseassociated brain changes in older adults. Frontiers in Aging Neuroscience, 2(23), 1-10. doi: 10.3389/fnagi.2010.00023

Cassilhas, R. C., Viana, V. A., Grassmann, V., Santos, R. T., Santos, R. F., Tufik, S., \& Mello, M. T. (2007). The impact of resistance exercise on the cognitive function of the elderly. Medicine and Science in Sports and Exercise, 39(8), 1401-1407. doi: $10.1249 / \mathrm{mss}$.obo13e318060111f

Cavanna, A. E., \& Trimble, M. R. (2006). The precuneus: A review of its functional anatomy and behavioural correlates. Brain, 129(3), 564-583.

doi:10.1093/brain/awloo4
Colcombe, S. J., Erickson, K. I., Scalf, P. E., Kim, J. S., Prakash, R., McAuley, E., ... \& Kramer, A. F. (2006). Aerobic exercise training increases brain volume in aging humans. The Journals of Gerontology Series A: Biological Sciences and Medical Sciences, 61(11), 1166-1170. doi: 10.1093/gerona/61.11.1166

Colcombe, S., \& Kramer, A. F. (2003). Fitness effects on the cognitive function of older adult: A meta-analytic study. Psychological Science, 14(2), 125-130. doi: 10.1111/1467-928o.to1-1-01430

Cotman, C. W., \& Berchtold, N. C. (2002). Exercise: A behavioral intervention to enhance brain health and plasticity. Trends in Neurosciences, 25(6), 295301. doi:10.1016/So166-2236(02)02143-4

Coutureau, E., \& Di Scala, G. (2009). Entorhinal cortex and cognition. Progress in Neuro-Psychopharmacology and Biological Psychiatry, 33(5), 753-761. doi:10.1016/j.pnpbp.2009.03.038

Davis, J. C., Marra, C. A., Beattie, B. L., Robertson, M. C., Najafzadeh, M., Graf, P., ... \& Liu-Ambrose, T. (2010). Sustained cognitive and economic benefits of resistance training among community-dwelling senior women: A 1-year follow-up study of the Brain Power study. Archives of Internal Medicine, 170(22), 2036-2038. doi: 10.1001/archinternmed.2010.462

Deary, I. J., Corley, J., Gow, A. J., Harris, S. E., Houlihan, L. M., Marioni, R. E., ... \& Starr, J. M. (2009). Ageassociated cognitive decline. British Medical Bulletin, 92(1), 135-152. doi: 10.1093/bmb/ldpo33

de Melo Coelho, F. G., Gobbi, S., Andreatto, C. A. A., Corazza, D. I., Pedroso, R. V., \& Santos-Galduróz, R. F. (2013). Physical exercise modulates peripheral levels of brain-derived neurotrophic factor (BDNF): A systematic review of experimental studies in the elderly. Archives of Gerontology and Geriatrics, 56(1), 10-15. doi: 10.1016/j.archger.2012.06.003

Diamond, A. (2013). Executive functions. Annual review of psychology, 64(1), 135-168. doi:10.1146/annurevpsych-113011-143750

Erickson, K. I., Raji, C. A., Lopez, O. L., Becker, J. T., Rosano, C., Newman, A. B., ... \& Kuller, L. H. (2010). Physical activity predicts gray matter volume in late adulthood: The Cardiovascular Health Study. Neurology, 75(16), 1415-1422. doi: 10.1212/WNL.obo13e3181f88359 
Erickson, K. I., Voss, M. W., Prakash, R. S., Basak, C., Szabo, A., Chaddock, L., ... \& Kramer, A. F. (2011). Exercise training increases size of hippocampus and improves memory. Proceedings of the National Academy of Sciences, 108(7), 3017-3022.

doi:10.1073/pnas.1015950108

Etnier, J. (2009). Chronic exercise and cognition in older adults. In T. McMorris, P.D.Tomporowski \& M. Audifffren (Eds.), Exercise and Cognition (pp. 227248).

Hoboken, NJ: Wiley-Blackwell.Fukuda, K., Vogel, E., Mayr, U., \& Awh, E. (2010). Quantity, not quality: The relationship between fluid intelligence and working memory capacity. Psychonomic Bulletin \& Review, 17(5), 673-679. doi: 10.3758/17.5.673

Grant, D. A., \& Berg, E. (1948). A behavioral analysis of degree of reinforcement and ease of shifting to new responses in a Weigl-type card-sorting problem. Journal of experimental psychology, 38(4), 404-411. doi:10.1037/hoo59831

Hatta, A., Nishihira, Y., \& Higashiura, T. (2013). Effects of a single bout of walking on psychophysiologic responses and executive function in elderly adults: A pilot study. Clinical Interventions in Aging, 8, 945952. doi: $10.2147 /$ CIA.S46405

Johnson, N. F., Kim, C., Clasey, J. L., Bailey, A., \& Gold, B. T. (2012). Cardiorespiratory fitness is positively correlated with cerebral white matter integrity in healthy seniors. Neuroimage, 59(2), 1514-1523. doi: $10.1016 / j$.neuroimage.2011.08.032

Liu-Ambrose, T., Nagamatsu, L. S., Graf, P., Beattie, B. L., Ashe, M. C., \& Handy, T. C. (2010). Resistance training and executive functions: A 12-month randomized controlled trial. Archives of Internal Medicine, 170(2), 170-178. doi: 10.1001/archinternmed.2009.494

Marmeleira, J. F., Godinho, M. B., \& Fernandes, O. M. (2009). The effects of an exercise program on several abilities associated with driving performance in older adults. Accident Analysis \& Prevention, 41(1), 90-97. doi: 10.1016/j.aap.2008.09.008
Mortimer, J. A., Ding, D., Borenstein, A. R., DeCarli, C., Guo, Q., Wu, Y., ... \& Chu, S. (2012). Changes in brain volume and cognition in a randomized trial of exercise and social interaction in a communitybased sample of non-demented Chinese elders. Journal of Alzheimer's Disease, 30(4), 757-766. doi: 10.3233/JAD-2012-120079

Muscari, A., Giannoni, C., Pierpaoli, L., Berzigotti, A., Maietta, P., Foschi, E., ...\&Zoli, M. (2010). Chronic endurance exercise training prevents aging-related cognitive decline in healthy older adults: A randomized controlled trial. International Journal of Geriatric Psychiatry, 25(10), 1055-1064. doi: $10.1002 / g p s .2462$

Mussolino, M. E., \& Gillum, R. F. (2008). Low bone mineral density and mortality in men and women: The Third National Health and Nutrition Examination Survey linked mortality file. Annals of Epidemiology, 18(11), 847-850. doi: 10.1016/j.annepidem.2008.07.003

Nagamatsu, L. S., Handy, T. C., Hsu, C. L., Voss, M., \& LiuAmbrose, T. (2012). Resistance training promotes cognitive and functional brain plasticity in seniors with probable mild cognitive impairment. Archives of Internal Medicine, 172(8), 666-668. doi: 10.1001/archinternmed.2012.379

Nithianantharajah, J., \& Hannan, A. J. (2009). The neurobiology of brain and cognitive reserve: mental and physical activity as modulators of brain disorders. Progress in Neurobiology, 89(4), 369-382. doi: 10.1016/j.pneurobio.2009.10.001

Oken, B. S., Zajdel, D., Kishiyama, S., Flegal, K., Dehen, C., Haas, M., ... \& Leyva, J. (2006). Randomized, controlled, six-month trial of yoga in healthy seniors: Effects on cognition and quality of life. Alternative Therapies in Health and Medicine, 12(1), 40-47. Retrieved from: http://www.alternativetherapies.com/

Rasmussen, C. (2005). Executive functioning and working memory in fetal alcohol spectrum disorder. Alcoholism: Clinical and Experimental Research, 29(8), 1359-1367. doi: 10.1097/01.alc.0000175040.91007.do 
Rovio, S., Kåreholt, I., Helkala, E. L., Viitanen, M., Winblad, B., Tuomilehto, J., ... \& Kivipelto, M. (2005). Leisure-time physical activity at midlife and the risk of dementia and Alzheimer's disease. The Lancet Neurology, 4(11), 705-711. doi:

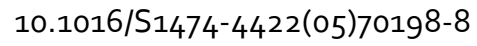

Rovio, S., Spulber, G., Nieminen, L. J., Niskanen, E., Winblad, B., Tuomilehto, J., ... \& Kivipelto, M. (2010). The effect of midlife physical activity on structural brain changes in the elderly.

Neurobiology of Aging, 31(11), 1927-1936. doi: 10.1016/j.neuobiolaging.2008.10.007

Rubenstein, L. Z. (2006). Falls in older people: epidemiology, risk factors and strategies for prevention. Age and Ageing, 35(suppl 2), ii37ii41.doi: 10.1093/ageing/aflo84

Sibley, B. A., \&Etnier, J. L. (2003). The relationship between physical activity and cognition in children: a metaanalysis. Pediatric Exercise Science, 15(3), 243-256. Retrieved from: http://journals.humankinetics.com/pes

Smiley-Oyen, A. L., Lowry, K. A., Francois, S. J., Kohut, M. L., \&Ekkekakis, P. (2008). Exercise, fitness, and neurocognitive function in older adults: the "selective improvement" and "cardiovascular fitness" hypotheses. Annals of Behavioral Medicine, 36(3), 280-291. doi: 10.1007/s12160-008-9064-5

Song, Y., \& Hakoda, Y. (2014). Executive and non-executive functions in attention deficit hyperactivity disorder of the inattentive type (ADHD-I): a cognitive profile. Journal of Behavioral and Brain Science, 2014. doi: 10.4236/jbbs.2014.41001

Stern, Y. (2002). What is cognitive reserve? Theory and research application of the reserve concept. Journal of the International Neuropsychological Society, 8(03), 448-46o. doi: $10.1017 / \mathrm{s} 1355617702813248$

Stroth, S., Reinhardt, R. K., Thöne, J., Hille, K., Schneider, M., Härtel, S., ... \& Spitzer, M. (2010). Impact of aerobic exercise training on cognitive functions and affect associated to the COMT polymorphism in young adults. Neurobiology of Learning and Memory, 94(3), 364-372. doi:10.1016/j.nlm.2010.08.003
Tomporowski, P. D. (2003). Effects of acute bouts of exercise on cognition. Acta Psychologica, 112(3), 297-324. doi:10.1016/Sooo1-6918(02)00134-8

Tseng, B. Y., Gundapuneedi, T., Khan, M. A., Diaz-Arrastia, R., Levine, B. D., Lu, H., ... \& Zhang, R. (2013). White matter integrity in physically fit older adults. Neuroimage, 82, 510-516. doi: 10.1016/j.neuroimage.2013.06.011

Tucker, A. M., \& Stern, Y. (2011). Cognitive reserve in aging. Current Alzheimer Research, 8(4), 354-36o. doi: $10.2174 / 156720511795745320$

Vogel, T., Brechat, P. H., Leprêtre, P. M., Kaltenbach, G., Berthel, M., \& Lonsdorfer, J. (2009). Health benefits of physical activity in older patients: $A$ review. International Journal of Clinical Practice, 63(2), 303-320. doi: 10.1111/j.17421241.2008.01957.x

Voss, M. W., Prakash, R. S., Erickson, K. I., Basak, C., Chaddock, L., Kim, J. S., ... \& Kramer, A. F. (2010). Plasticity of brain networks in a randomized intervention trial of exercise training in older adults. Frontiers in Aging Neuroscience, 2, 1-17. doi:10.3389/fnagi.2010.00032

Warburton, D. E., Nicol, C. W., \&Bredin, S. S. (2006). Health benefits of physical activity: The evidence. Canadian Medical Association Journal, 174(6), 8018og. doi: 10.1503/cmaj.051351

Williamson, J. D., Espeland, M., Kritchevsky, S. B., Newman, A. B., King, A. C., Pahor, M., ...\& Miller, M. E. (2009). Changes in cognitive function in a randomized trial of physical activity: Results of the lifestyle interventions and independence for elders pilot study. The Journals of Gerontology Series A: Biological Sciences and Medical Sciences, $64 A(6)$, 688-694. doi: 10.1093/gerona/glpo14

World Health Organization (2015).Physical Activity and Adults. Retrieved from http://www.who.int/dietphysicalactivity/factsheet_ adults/en/ 
